\title{
The Association between Oral Hygiene and Gastric Pathology in Patients with Dyspepsia: a Cross-Sectional Study in Southeast Iran
}

\author{
Leili Zahedi ${ }^{1}$, Elham Jafari ${ }^{*}$, Molook Torabi Parizi ${ }^{3}$, Sara Shafieipour ${ }^{4}$, Mehdi Hayat Bakhsh Abbasi ${ }^{5}$, \\ Sodaif Darvish Moghadam ${ }^{6}$, Mohammad Javad Zahedi ${ }^{6}$
}

1. Researcher, Kerman Oral and Dental Diseases Research Center, Kerman University of Medical Sciences, Kerman, Iran

2. Assistant Professor, Pathology and stem cells Research Center, Kerman University of Medical Science ,Kerman,Iran

3. Associate Professor, Kerman Oral and Dental Diseases Research Center, Kerman University of Medical Sciences, Kerman, Iran

4. Assistant Professor of Internal Medicine, Digestive Research Center, Kerman University of Medical Sciences, Kerman, Iran

5. Associate Professor of Internal Medicine, Digestive Research Center, Kerman University of Medical Sciences, Kerman, Iran

6. Professor of Internal Medicine, Digestive Research Center, Kerman University of Medical Sciences, Kerman, Iran

\section{* Corresponding Author:}

Elham Jafari, MD

Pathology and stem cells research center, Kerman University of Medical Science, Kerman,Iran.

Telefax: + 983432223066

Email: ejfarda@yahoo.com

Received: 03 Sep. 2016

Accepted: 11 De. 2016

\author{
ABSTRACT
}

\section{BACKGROUND}

Many studies have reported an association between periodontal infections and some systemic diseases such as respiratory and cardiovascular diseases. Some studies found a direct association between chronic gastritis caused by Helicobacter pylori (HP) infection and poor periodontal health.

\section{METHODS}

In a cross-sectional study from November 2014 to December 2015 in Kerman, the largest province in southeast Iran, patients with dyspepsia who were candidate for diagnostic upper gastrointestinal (GI) endoscopy were included in our study. Decayed, Missing, and Filled Teeth (DMFT) index and Loe plaque index that are two popular indexes in dental epidemiology were used to assess the oral health by a dentist before the upper GI endoscopy. According to the Loe plaque index, score: $0=$ no plaque, score: $1=$ a film of plaque attaching to the free gingival border and near area of the tooth, score: $2=$ moderate reposition of deposits within the gingival pocket, score $: 3=$ plenty of soft matter within the gingival pocket \pm on the tooth and gingival border. Scores $\leq 1$, 2 and 3 equal to good, moderate, and poor oral hygiene, respectively. During upper GI endoscopy a total of six biopsy samples were taken from fundus, body, and antrum. A pathologist reported these samples according to Sidney's classification into superficial gastritis, atrophic gastritis, intestinal metaplasia, and dysplasia.

\section{RESULTS}

According to Sidney's classification 77 (89.5\%) patients had superficial gastritis, $3(3.5 \%)$ had atrophic gastritis, and $6(7 \%)$ had intestinal metaplasia. HP was found in $80.2 \%$ of the gastric mucosal biopsy samples. There were not statistically significant relationship between Sidney's classification, presence of HP in gastric mucosal biopsies, and hygiene indicators $(p>0.05)$. No relation was found between the DMFT index and superficial gastritis, atrophic gastritis, and intestinal metaplasia $(p>0.05)$. Gastric infection with HP was found in $70 \%, 75 \%$, and $100 \%$ of patients with mild, moderate, and sever DMFT index, respectively.

\section{CONCLUSION}

Our study showed that there might be a relation between poor oral hygiene and gastric precancerous lesions. In addition, HP infection in gastric histopathology might be associated with periodontal disease.

KEYWORDS: Oralhygiene, Dyspepsia, Chronic gastritis.

Please cite this paper as:

Zahedi L, Jafari E, Torabi Parizi M, Shafiei pour S, Hayat Bakhsh Abbasi M, DarvishMoghadam S, Zahedi MJ. The Association between Oral Hygiene and Gastric Pathology in Patients with Dyspepsia: a Cross-Sectional Study in Southeast Iran. Middle East J Dig Dis 2017;9:33-38. DOI: 10.15171/mejdd.2016.49

\section{INTRODUCTION}

Oral health is an important component of general health. ${ }^{1}$ Many studies have found an association between periodontal infection and some systemic 
diseasessuch as some respiratory and cardiovascular diseases. $^{2,3}$ So, increasing the awareness about oral health should be one of the healthprograms in order to reduce the outcomes of systemic diseases. ${ }^{4}$

Some studies found a direct association between chronic gastritis caused by Helicobacter pylori (HP) infection and poor periodontal health. HP is an opportunist gram negative microorganism that causes some gastrointestinal (GI) diseases in humans via infected foods and water. ${ }^{5}$ More than $50 \%$ of the general population worldwide have been infected by this organism and the prevalence of this infection in Iran seems to be $90 \% .^{6,7}$ This infection is usually asymptomatic and associated with superficial gastritis, which is often reversible by appropriate anti HP treatment.But persistent infection causes chronic gastritis that can progress to atrophic gastritis, intestinal metaplasia, dysplasia, and ultimately gastric adenocarcinoma. ${ }^{6,8} \mathrm{HP}$ is present in saliva, tongue dorsum, and dental plaque as a natural flora.It seems that mouth cavity is a suitable reservoir for recurrence of gastric HPinfection. ${ }^{9}$

Poor dental and oral hygiene such as negligence in teeth brushing can be a risk factor for the presence of HP in oral cavity. ${ }^{10,11}$ Rarely, HP in dental plaque is eliminated by anti HPeradication therapy, and this may play a role as a source for future reinfection. Therefore, eradication of HP from dental plaques and oral cavity should be an important part of the comprehensive management of HP-associated GI diseases. ${ }^{2-15}$

Up to now, the association between gastric pathology and oral hygiene has been reported by limited studies. ${ }^{16-18}$ Salazar and colleagues showed that about $70 \%$ of patients with precancerous lesionsin gastric biopsy had poor oral hygiene. ${ }^{16}$ In 2014, Boylanand co-workers in a study of 262 patients found that periodontal disease was associated with increased risk of peptic ulcer disease. ${ }^{17}$ So, the purpose of this study was to assess the association between oral health and gastric pathology as the first study in Iran.

\section{MATERIALS AND METHODS}

\section{Patients' population}

In a cross-sectional study from November 2014 to December 2015 in Kerman, the largest province in southeast Iran, patients with dyspepsia who were candidate for diagnostic upper GI endoscopy and were referred to a gastroenterology clinic in AfzalipourHospital were included in our study. Inclusion criteria were dyspeptic patients withepigastric pain or discomfort who had history of anti-dyspeptic therapy without remission and onset of dyspepsia after 50 years old.

Exclusion criteria were: patient with history ofdiabetes mellitus, cirrhosis, renal failure, cancer, and immunosuppressive drug consumption.

Demographic data from all the patients were collected from their medical records and entered to a checklist.

Decayed, Missing, and Filled Teeth (DMFT) index and Loe plaque index that are two popular indexes in dental epidemiology were used to assessthe oral health by a dentist before the upper GI endoscopy.In order to determine DMFT index, the examination method was based on WHO criteria. That is, the patient sits in dental unitand by flat dental mirror and ordering light he/she is assessed for number of extracted teeth due to decayrestored teeth and decayed teeth. Dental plaque measuring index used in this research was Loa index. According to the Loe plaque index, score $0=$ no plaque,score: $1=$ a film of plaque attaching to the free gingival border and near area of the tooth,score $2=$ moderate reposition of deposits within the gingival pocket,score $3=$ plenty of soft matter within the gingival pocket \pm on the tooth and gingival border. Scores $\leq 1,2$, and 3 equal to good, moderate, and poor oral hygiene, respectively. ${ }^{19}$

We used $\mathrm{SiC}$ index for dividing patients into two groups according to their DMFT. For this purpose we chose the one third of the population with the highest DMFT values. The SiC Index is the one third of the study group with the highest DMFT score ${ }^{20}$ and the other two third have mild to moderate DMFT.

During upper GI endoscopy a total of six biopsy samples were taken from fundus, body, and antrum.After tissue processing a pathologist who was notaware about the patients' oral health,reported these samples according to Sidney's classification into superficial gastritis, atrophic gastritis, intestinal metaplasia, and dysplasia.

\section{Statistical analysis}

Chi-square and t tests were used for statisticalanalysis. SPSS software version 20 was used for data analysis. $p$ value $<0.05$ was considered as statistically significant. 
Ethics:

The protocol of our study was approved by EthicsCommittee of Kerman University of Medical Sciences. All the participants signed a consent form and their data were kept secret.

\section{RESULTS}

86 patients with dyspepsia consisted of 34 (39.5\%) women and $52(60.5 \%)$ men were included in our study. The mean age of the patients was 40.32 years. The youngest and the oldest patients were 20 and 63 years old, respectively.

The patients were evaluated considering their education: $7 \%$ were illiterate, $27.9 \%$ were at primary school level,26.7\% had high school diploma, and 38.4\% had higher education. The demographic information about sex and education are shown in table 1.

The characteristics of the patients including: hygiene indicators (the number of teeth brushing per day, flossing, and visiting the dentist), DMFT and,Loe plaque index are summarized in tables 2, and 3 .

According to Sidney's classification 77 (89.5\%) patients had superficial gastritis, 3 (3.5\%) had atrophic gastritis, and $6(7 \%)$ had intestinal metaplasia. HP was found in $80.2 \%$ of the gastric mucosal biopsy samples. There were not statistically significant relationship between Sidney's classification, presence of HP in gastric mucosal biopsies, and hygiene indicators $(p>0.05)$. The relationship between gastritis degree andexistence of HP, and also the relationship between DMFT, Plaque index, and HP colonization amount are summarized in tables 4 , and 5, respectively.

There were not statistically significant associations between Loe plaque index and superficial gastritis and intestinal metaplasia $(p=0.825)$. In contrary, there was a statistically significant relationship between Loe plaque index, and the presence of atrophic gastritis in gastric mucosal biopsies ( $p=0.001$, table 3 ). Also, the relation between Loe plaque index and finding of HP in gastric mucosal biopsies was statistically significant ( $p$.value $<0.05)$. HP was found in $66 \%, 82.6 \%$, and $100 \%$ of the patients with good, moderate, and poor Loe plaque index, respectively.

No relation was found between the DMFT index and superficialgastritis, atrophic gastritis, and intestinal
Table 1: Demographic data

\begin{tabular}{llc}
\hline Variables & Male & $\begin{array}{c}\text { Frequency } \\
\text { (percent) }\end{array}$ \\
\hline \multirow{2}{*}{ Sex } & Female & $52(60.5)$ \\
\hline \multirow{2}{*}{ Education } & Illiterate & $34(39.5)$ \\
\cline { 2 - 3 } & Primary school & $6(7)$ \\
\cline { 2 - 3 } & High school & $24(27.9)$ \\
\cline { 2 - 3 } & Higher Education & $23(26.7)$ \\
\hline $\begin{array}{l}\text { Age mean } \\
\text { (standadrd deviation })\end{array}$ & 40(13.2) & \\
\hline
\end{tabular}

Table 2: Frequency of oral hygiene indices

\begin{tabular}{llc}
\hline \multirow{2}{*}{ Variables } & \multicolumn{1}{c}{$\begin{array}{c}\text { Frequency } \\
\text { (percent) }\end{array}$} \\
\hline \multirow{2}{*}{$\begin{array}{l}\text { Tooth brushing times } \\
\text { per day }\end{array}$} & No brushing & $15(17.4)$ \\
\cline { 2 - 3 } & Irregular & $25(29.06)$ \\
\cline { 2 - 3 } Dental floss usage & Twice & $30(34.9)$ \\
\hline \multirow{2}{*}{ Last visiting a dentist } & No & $16(18.6)$ \\
\cline { 2 - 3 } & Never & $16(18.6)$ \\
\cline { 2 - 3 } & 6 month ago & $70(81.4)$ \\
\cline { 2 - 3 } & A year ago & $27(31.4)$ \\
\cline { 2 - 3 } & More than one year ago & $31(36)$ \\
\hline
\end{tabular}

Table 3: Frequency of dental \&gingival indices

\begin{tabular}{lll}
\hline \multirow{2}{*}{ DMFT index } & SIC & $20(23.3)$ \\
\cline { 2 - 3 } & Other 2/3 & $66(76.7)$ \\
\hline \multirow{2}{*}{ Loe index } & Good & $39(45.3)$ \\
\cline { 2 - 3 } & Moderate & $23(26.7)$ \\
\cline { 2 - 3 } & Poor & $24(27.9)$ \\
\hline
\end{tabular}

metaplasia $(p>0.05)$.

Gastric infection with HP was found in $70 \%, 75 \%$, and $100 \%$ of the patients with mild,moderate, and sever DMFT index, respectively.

There was nostatistically meaningful difference based on education between the illiterate individuals, primary school level,high school diploma, and higher education regarding the various degrees of HP.Also the difference between the frequency of HP degrees in stomach was not meaningful based on age and sex $(p>0.05)$. 
Table 4: The relationship between digree of gastritis and H.pylori

\begin{tabular}{lccccccc}
\hline & \multicolumn{9}{c}{ H.pylori } & & \\
\hline & No & Mild & Moderate & Sever & Total & P value \\
\hline Superficial gastritis & $17(21.5)$ & $24(30.4)$ & $28(35.4)$ & $10(12.7)$ & 79 & \\
\hline Intestinal metaplasia & $0(0)$ & $0(0)$ & $3(50)$ & $3(50)$ & 6 & 0.088 \\
\hline Gastric atrophy & $0(0)$ & $1(100)$ & $0(0)$ & $0(0)$ & 1 & \\
\hline
\end{tabular}

Table 5: The relationship between DMFT and Plaque indexes with gastric $H$.pylori grades

\begin{tabular}{|c|c|c|c|c|c|c|}
\hline \multicolumn{7}{|c|}{ H.pylori } \\
\hline & & Absent (no H.pylori) & Mild & Moderate & Sever & P value \\
\hline \multirow{2}{*}{ DMFT } & Mild to moderate & $17(100)$ & $25(100)$ & $16(51.6)$ & $8(61.5)$ & \multirow{2}{*}{0.001} \\
\hline & sever & $0(0)$ & $0(0)$ & $15(48.4)$ & $5(38.5)$ & \\
\hline \multirow{3}{*}{ Plaque Index } & good & $13(76.5)$ & $17(68)$ & $7(22.6)$ & $2(15.4)$ & \multirow{3}{*}{0.001} \\
\hline & moderate & $4(23.5)$ & $8(32)$ & $9(29)$ & $2(15.4)$ & \\
\hline & bad & $0(0)$ & $0(0)$ & $15(48.4)$ & $9(69.2)$ & \\
\hline
\end{tabular}

\section{DISCUSSION}

The aim of our study was to assess the association between oral health and gastric pathology. Due to the best of our knowledge, up to now, there was no research from Iran in this regard in the literature.

Our study showed that there was a direct relationship between the presence of HP in the stomach and unsatisfactory dental and gingival indices. Our data are consistent with the literature that suggests oral cavity and oral plaque may be a reservoir for HP leading to the recurrence of gastric infection. ${ }^{9-15}$ in 2014, Wang and colleaguesreported that simultaneous treatment of periodontal disease and oral HP could increase the success rate of the eradication of the gastric HP infection from $61.33 \%$ to $82.26 \% .{ }^{21}$ In addition, Zaricand co-workers indicated that $77.3 \%$ of the patients treated with combined periodontal disease and anti-HP triple therapy showed successful eradication of gastric HPcompared with $47.6 \%$ who underwent triple therapy alone. ${ }^{22}$

There were an association between the unpleasant Loe plaque index and atrophic gastritis in our study. Atrophic gastritis is the main precursor lesion of gastric cancer, and HP infection is the most important cause of atrophic gastritis. ${ }^{26}$ Periodontal disease is associated with alteration in oral microbiome. ${ }^{17}$ Many studies have shown higher levels of HP in periodontitis status than in normal periodontal condition. ${ }^{25-27}$ On the other hand periodontal pathogens and disease are associated with high levels of inflammatory cytokinesthat lead to induction of systemic chronic inflammation. ${ }^{18}$

Alasqah research indicated that in patients with chronic periodontitis, the prevalence of HP existence in stomach was higher than patients without periodontitis. ${ }^{23}$ Beliand colleagues in their study on 124 patients with or without gastric HP infection,reported meaningful relationship between HP infection and patients'periodental status, but there was no meaningful relationship between the number of teeth and HP infection. ${ }^{24}$ In contrast, Berroteranand co-workers found no relationship between HP infection and periodontal disease (Loe and Sillness indices), dental caries, and dental hygiene. ${ }^{25}$ In this research,it was specified that periodontal diseases and DMFT indexhad meaningful relationship with gastric HP infection.Some studies have declared that shift in oral flora and systemic inflammation can affect some organs such as stomach, and pancreas ${ }^{28}$ and result in tumor development and growth. ${ }^{16}$

Boylanand colleagues indicated that risk of peptic ulcerincreased with the presence of periodontal disease. ${ }^{18}$ Salazar and co-workers found that among patients with higher grades of periodontal disease, periodontal pathogens that measured in plaque were related to gastric precancerous lesions attendance. ${ }^{16}$

Our study didnot show a relation between the DMFT index and gastric histopathology.It seems that the speed of bacterial colonization effect on teeth is much slower 
than periodontium. So it seems that, like periodontal conditions, the tooth condition cannot be associated with gastric pathology. Limited studies reported a positive association between poor DMFT index and the risk of gastric adenocarcinoma. ${ }^{30-33}$ Shakeri and co-workers in a study of 309 patients with gastric adenocarcinoma found a significant association between tooth loss and poor DMFT score,and the risk of gastric cancer. In addition apparent association was found only for gastric cardia cancer, for those subjects who brushed their teeth less than daily. ${ }^{31}$ In a prospective study in China, tooth loss, increased the risk of developing esophageal and gastric cancers. ${ }^{32}$ On the contrary, Salazar and colleagues didnot find such a relation in a study of 41 patients with gastric precancerous lesions including intestinal metaplasia or chronic atrophic gastritis. ${ }^{18}$ There was no meaningful relationship when evaluating the relationship between histopathological findings of gastric mucosa and oral hygiene indices including the times of brushing the teeth,using floss, and the last time visiting by the dentist.In Salazar's study,the patients without precancerous lesions of the stomach used more floss, but there was no such relationship when using toothbrush and times of using it. ${ }^{18}$

Tooth loss can be the outcome of many factors such as periodontal disease, caries, trauma, some systemic diseases, or low socioeconomic status, so For study about tooth loss such factors should be carefully considered.

Low sample size is the major limitation of our study and we suggest a larger study for better assessment of the association between periodontal disease and GI pathology.This research is one of the few studies that assess the association between oral health and gastric pathology.

In conclusion, our study showed that there might be a relation between poor oral hygiene andgastric precancerous lesions. In addition,HP in gastric histopathology was associated with periodontal disease.Because the HP in the mouth can transfer into stomach and even causes repeated gastric infection after treatments and removal. So periodontal treatments along with the treatment protocols of HP infection should be considered.

\section{CONFLICT OF INTEREST}

The authors declare no conflict of interest related to this work.

\section{REFERENCES}

1. Petersen PE. Global policy for improvement of oral health in the 21st century.Community Dent Oral Epidemiol 2009;37:1-8. Doi: 10.1111/j.1600-0528.2008.00448.x.

2. Haumschild MS, Haumschild RJ. The Importance of Oral Health in Long-Term Care. J Am Med Dir Assoc. 2009;10:667-71. doi: 10.1016/j.jamda.2009.01.002.

3. Alman AC, Johnson LR, Calverley DC, Grunwald GK, Lezotte DC, Harwood JE. Loss of alveolar bone due to periodontal disease exhibits a threshold on the association with coronary heart disease. J Periodontol 2011;82:130413. doi: 10.1902/jop.2011.100647.

4. Petersen PE. World Health Organization global policy for improvement of oral health-World Health Assembly 2007.Int Dent J 2008;58:115-21. doi: 10.1111/j.1875595X.2008.tb00185.x

5. Vale FF, Vítor JM. Transmission pathway of Helicobacter pylori: does food play a role in rural and urban areas?Int J Food Microbiol 2010;138:1-12. doi: 10.1016/j.ijfoodmicro.2010.01.016.

6. Crew KD, Neugut AI. Epidemiology ofgastric cancer. World J Gastroenterol 2006;12:354-62. doi: 10.3748/ WJG.v12.i3.354

7. Goh KL, Chan WK, Shiota S, Yamaoka Y. Epidemiology of Helicobacter pylori Infection and Public Health Implications. Helicobacter 2011;16Suppl 1:1-9. doi: 10.1111/j.1523-5378.2011.00874.x

8. Hosseini E, PoursinaF,Van de Wiele T, GhasemianSafaei $\mathrm{H}$,Adibi P. Helicobacter pylori in Iran: A systematic review on the association of genotypes and gastroduodenal diseases. J Res Med Sci 2012 ;17: 280-92. doi: 10.1111/j.1523-5378.2011.00892.x

9. Navabi N, Aramon M, MirzazadehA.Does the presence of the Helicobacter pylori in the dental plaque associate with its gastric infection? A meta-analysis and systematic review. Dent Res J (Isfahan) 2011;8:178-82. doi: 10.4103/1735-3327.86033.

10. Liu Y, Lin H, Bai Y, Qin X, Zheng X, Sun Y, et al. Study on the Relationship Between Helicobacter pylori in the Dental Plaque and the Occurrence of Dental Caries or Oral Hygiene Index. Helicobacter 2008;13:256-60. doi: $10.1111 / j .1523-5378.2008 .00602 . x$

11. Nouraie M, Latifi-Navid S, Rezvan H, Radmard A.R, Maghsudlu M, Zaer-Rezaii H, et al. Childhood Hygienic Practice and Family Education Status Determine the Prevalence of Helicobacter pylori Infection in Iran. Helicobacter 2009;14:40-6. DOI: 10.1111/j.1523-5378.2009.00657.x

12. Anand PS, NandakumarKa, Shenoy KT. Are Dental Plaque, Poor Oral Hygiene, and Periodontal Disease Associated With Helicobacter pylori Infection. J Periodontol 2006;77:692-8. doi:10.1902/jop.2006.050163

13. Gebara EC, Faria CM, Pannuti C, Chehter L, Mayer MP, Lima LA..Persistence of Helicobacter pylori in the oral cavity after systemic eradication therapy. $J$ ClinPeriodontol 2006;33:329-33. doi: 10.1111/j.1600- 
051X.2006.00915.X

14. Namiot DB, Namiot Z, Kemona A, Bucki R, Gotębiewska M. Oral Health Status and Oral Hygiene Practices of Patients with Peptic Ulcer and How These Affect Helicobacter pylori Eradication from the Stomach. Helicobacter 2007;12:63-7. doi: 10.1111/j.1523-5378.2007.00471.x

15. Gürbüz AK, Ozel AM, Yazgan Y, Celik M, Yildirim S. Oral colonization of Helicobacter pylori: risk factors and response to eradication therapy. South MedJ2003;96:2447. doi: 10.1097/01.SMJ.0000051069.50950.2B

16. Salazar CR, Francois F, Li Y, Corby P, Hays R, Leung $\mathrm{C}$, et al. Association between oral health and gastric precancerous lesions. Carcinogenesis 2011;33:399-403. doi: 10.1093/carcin/bgr284

17. Boylan MR, Khalili H, Huang ES, Michaud DS, Izard J, Joshipura K, et al. A Prospective Study of Periodontal Disease and Risk of Gastric and Duodenal Ulcer in Male Health Professionals. ClinTranslGastroenterol 2014;5:e49. doi: 10.1038/ctg.2013.14.

18. Salazar CR,Sun J, Li Y, Francois F, Corby P, Perez-Perez $\mathrm{G}$, et al. Association between selected oral pathogens and gastric precancerous lesions. PLoS One 2013;8:e51604. doi: 10.1371/journal.pone.0051604.

19. Rebelo MAB, De Queiroz AC. Gingival Indices: State of Art. INTECH Open Access Publisher.online: http://cdn. intechopen.com/pdfs-wm/20291.pdf.2011

20. Nishi M, Bratthall D, Stjernswärd J. How to calculate the significant caries index (SiC Index). WHO Collaborating Centre [online]. PDF Vers. 2001;1.

21. Wang XM, Yee KC,Hazeki-Taylor N, Li J, Fu HY, Huang ML, et al. Oral Helicobacter pylori, its relationship to successful eradication of gastric H. pylori and saliva culture conformation. J Physiol Pharmacol 2014;65:559-66.

22. Zaric S, Bojic B, JankovicLJ, Dapcevic B, Popovic B, Cakic S, et al. Periodontal therapy improves gastric Helicobacter pylori eradication. J Dent Res 2009;88: 946-50.

23. Al Asqah M, Al Hamoudi N, Anil S, Al Jebreen A, AlHamoudi WK. Is the presence of Helicobacter pylori in the dental plaque of patients with chronic periodontitis a risk factor for gastric infection? Can J Gastroenterol 2009;23:177-9. doi: 10.1155/2009/950527

24. Bali D, Rosamma J, Bali A. The Association of Dental Plaque And Helicobacter Pylori Infection In Dyspeptic Patients Undergoing Endoscopy. J Clin Diagn Res 2010;4:3614-21.

25. Berroteran A, Perrone M, Correnti M, Cavazza ME, Tombazzi C, Goncalvez R, et al. Detection of Helicobacter pylori DNA in the oral cavity and gastroduodenal system of a Venezuelan population. J Med Microbiol 2002;51:764-70. doi: 10.1099/0022-1317-51-9-764

26. Park YH,Kim N. Review of atrophic gastritis and intestinal metaplasia as a premalignant lesion of gastric cancer. J Cancer Prev 2015;20:25-40. doi:10.15430/ JCP.2015.20.1.25
27. Anand PS, Kamath KP, Anil S. Role of dental plaque, saliva and periodontal disease in Helicobacter pylori infection. World J Gastroenterol 2014;20:5639-53. doi: 10.3748/WJG.v20.i19.5639

28. Sambashivaiah S, Bilichodmath S, Nanjaiah N, Kulal R. Helicobacter Pylori in periodontal pockets of chronic periodontitis patients with and without type II diabetes mellitus: a randomized controlled trial. Microbiol Res 2011;2(12). doi: 10.4081/mr.2011.e12

29. Silva DG, Stevens RH, Macedo JM, Albano RM, Falabella ME, Fischer RG, et al. Presence of Helicobacter pylori in supragingival dental plaque of individuals with periodontal disease and upper gastric diseases. Arch Oral Biol 2010;55:896-901. doi: 10.1016/j.archoralbio.2010.06.018.

30. Fitzpatrick SG, Katz J. The association between periodontal disease and cancer: A review of the literature. $J$ Dent 2010;38:83-95. doi: 10.1016/j.jdent.2009.10.007.

31. Shakeri R, Malekzadeh R, Etemadi A, Nasrollahzadeh D, AbediArdekani B, Khoshnia M, et al. Association of tooth loss and oral hygiene with risk of gastric adenocarcinoma. Cancer Prev Res (Phila)2013;6:477-82. doi: 10.1158/1940-6207.CAPR-12-0491

32. Abnet CC, Qiao YL, Mark SD, Dong ZW, Taylor PR, Dawsey SM. Prospective study of tooth loss and incident esophageal and gastric cancers in China.Cancer Causes Control 2001;12:847-54. doi: 10.1023/A:1012290009545

33. Abnet CC,Kamangar F, Dawsey SM, Stolzenberg-Solomon RZ, Albanes D, Pietinen P, et al. Tooth loss is associated with increased risk of gastric non-cardia adenocarcinoma in a cohort of Finnish smokers. Scand J Gastroenterol 2005;40:681-7. doi: 10.1080/00365520510015430 